\title{
Cortical activation during rhythmic hand movements performed under three types of control: An fMRI study
}

\author{
R. A. BERNARD, D. A. GORAN, S. T. SAKAI, T. H. CARR, and D. MCFARLANE \\ Michigan State University, East Lansing, Michigan \\ B. NORDELL \\ Karolinska Hospital and Institute, Stockholm, Sweden
}

and

T. G. COOPER and E. J. POTCHEN

Michigan State University, East Lansing, Michigan

\begin{abstract}
Echoplanar fMRI was used to measure changes in cortical activation during the performance of a simple hand movement task under three types of voluntary control. Each of three imaging series alternated a task with rest: passive (in which the experimenter moved the hand), voluntary against low resistance, and voluntary against higher resistance. Contralateral activation was observed in the supplementary motor area (SMA), the primary motor cortex (M1), and the somatosensory cortex (S1) in all three tasks in each subject, whereas ipsilateral activation differed in each cortical region for each task. SMA had the widest prevalence of ipsilateral activation in all three tasks. In the M1, ipsilateral activation was observed in all but 1 subject in the two voluntary tasks but in only a few subjects in the S1 in any of the tasks. Quantitative changes in signal intensity and spatial extent of activation differentiated the voluntary tasks from the passive task and were most pronounced in the S1.
\end{abstract}

In a traditional, simplified view of motor cortical function, the supplementary motor area (SMA) plans motor acts, and the primary motor cortex (M1) executes the plan (Ghez \& Krakauer, 2000), whereas the somatosensory cortex (S1) gathers sensory feedback to evaluate the fidelity of the movement against the plan's specifications (Flament \& Hore, 1988; Prud'homme, Cohen, \& Kalaska, 1994; Soso \& Fetz, 1980).

Consistent with this view, single-unit recording studies in awake monkeys report that the activity of M1 cells is tightly coupled to a motor task and to movement parameters, such as force (Evarts, 1968; Evarts, Fromm, Kroller, \& Jennings, 1983; Georgopoulos, Ashe, Smyrnis, \& Taira, 1992; Hepp-Reymond, Wyss, \& Anner, 1978). However, Tanji and Kurata (1982) have reported that cells in the SMA also change activity during movement, suggesting that the SMA is also involved in movement execution. In addition, the role of the SMA in movement preparation is

This work was supported by the Department of Radiology at Michigan State University. We thank the students of Physiology 475, a laboratory course for undergraduate physiology majors at Michigan State University, who served as enthusiastic subjects in these experiments. Address correspondence to R. A. Bernard, Department of Physiology, Michigan State University, East Lansing, M1 48824 (e-mail: bernard@ msu.edu). based, in part, on the finding that a substantial portion of SMA cells fire prior to movement (Evarts \& Tanji, 1974). However, a population of M1 cells also fires prior to movement (Alexander \& Crutcher, 1990; Donoghue, Sanes, Hatsopoulos, \& Gaal, 1998), suggesting that the M1, along with the SMA, has a role in movement preparation.

Although it is accepted that the S1 provides somatosensory feedback to guide movements, it has been reported that cells in the M1, as well as the S1, are active during both passive and active movements (Fetz, Finocchio, Baker, \& Soso, 1980; Flament \& Hore, 1988; Lemon \& Porter, 1976; Prud'homme et al., 1994; Soso \& Fetz, 1980). This suggests that the need for control of execution, which is present for active movement but is lessened or eliminated for passive movement, does not fully determine the conditions of activation for the M1 or fully discriminate between conditions of activation for the $\mathrm{M} 1$ and the $\mathrm{S} 1$. In addition, cells in the SMA are also reported to respond to passive movements (Tanji, 1994). Thus, functional specialization within the motor control system is more complicated than is traditionally presented.

This functional complexity observed in awake monkeys is consistent with the anatomical finding that all these cortical areas receive thalamic afferents originating from skin, muscle, and joint receptors, the basal ganglia, and the cerebellum (Jones, 1985; Rouiller, Liang, Babalian, Moret, \& 
Wiesendanger, 1994; Sakai, Inase, \& Tanji, 1996; Schell $\&$ Strick, 1984). In addition, these areas are richly interconnected with each other through extensive ipsilateral and contralateral corticocortical projections (Jones, Coulter, $\&$ Hendry, 1978) and are known to give rise to corticospinal projections (Galea \& Darian-Smith, 1994; Murray \& Coulter, 1981).

Functional imaging studies of humans have also examined the role of the M1, S1, and SMA in a variety of motor tasks. Activation of the sensorimotor cortex (the M1 plus the S1) and activation of the SMA during performance are both widely reported (Colebatch, Deiber, Passingham, Friston, \& Frackowiak, 1991; Deiber et al., 1991; Grafton, Woods, \& Mazziotta, 1993; Porro et al., 1996; Turner, Grafton, Votaw, DeLong, \& Hoffmann, 1998). However, not all the studies reporting activation in the sensorimotor cortex have distinguished between the M1 and the S1, as is standardly done in the single-unit recording studies of awake monkeys. Where the distinction has been made, activation of the M1 has been consistently reported during voluntary movement, but activation of the $\mathrm{S} 1$ has not always been observed (Rao et al., 1993; Xiong, Parsons, Gao, \& Fox, 1999). Among studies in which voluntary movement has been compared with passive movement, two have reported activation of the S1 to the same degree in both types of movement but activation of the M1 only in the voluntary movement task (Mima et al., 1999; Rausch, Spengler, \& Eysel, 1998). A third study (Weiller et al., 1996) found that the M1 and the S1 were both activated, and to the same degree, during voluntary and passive movements. Thus, activation of the M1 and the S1 during voluntary and passive movements remains unclear in the functional imaging studies of humans.

Functional imaging studies on the effect of force on cortical activation during movement have also produced mixed results. Dettmers and others (Dettmers et al., 1996; Dettmers et al., 1995) reported increasing activation of the S1, M1, and SMA as a function of force exerted during a finger-tapping task. Another functional imaging study in which a sustained finger flexion task was used reported activation in the sensorimotor cortex as a function of exerted force (Thickbroom, Phillips, Morris, Byrnes, \& Mastaglia, 1998). However, no force-related changes were reported when a finger flexion task was used in two other functional imaging studies (Ludman, Cooper, PloutzSynder, Potchen, \& Meyer, 1996; Thickbroom et al., 1999).

It seems likely that the variability of the functional imaging results is due, in part, to the variety of motor tasks that have been used, ranging from elbow to individualfinger movements, with different ranges of motion and velocities, with different rates of movement, with active and passive control, and requiring various degrees of force. Furthermore, the results of functional imaging, which measures signals related to the hemodynamics of the metabolic demands of neural activity, have only infrequently been compared with the results of single-unit recording and have rarely been interpreted in light of emerging knowledge about how these two indicators of neural activity are re- lated (Bandettini \& Ungerleider, 2001; Heeger \& Ress, 2002; Logothetis, Paulis, Augath, Trinath, \& Oeltermann, 2001; Menon, 2001). Thus, a coherent picture of the neural substrate for motor control-especially in humans, where the functional imaging literature plays such a large rolewould seem to benefit from further study.

Accordingly, we undertook the present study, using fMRI to obtain a clearer picture of the cortical activation involved in a simple movement task in which joint position and displacement could be kept relatively constant while the type or degree of control required was varied. The chosen task consisted of rhythmic flexion of all the fingers of the right hand together, except the thumb, at a constant rate. Passive movement of the hand by the experimenter represented external, or minimal internal, control. The subject's voluntary movement of the hand against lesser versus greater resistance represented intermediate and greater amounts of internal control, allowing examination of sensitivity to variations in force.

Our specific goal was to determine how and to what extent activation in the M1, S1, and SMA, together and separately, was involved in the control of a simple movement. To do this, we manually identified the areas of interest in each individual brain, using commonly accepted anatomical landmarks, and measured the intensity and spatial extent of activation within each area. We then related our functional imaging findings to what is known regarding the connectivity and physiology of motor cortical areas in nonhuman primates.

\section{METHOD}

\section{Subjects}

Six male and 6 female volunteers from the student population of Michigan State University participated. They ranged in age from 18 to 23 years. All identified themselves as right-handed. All gave informed consent to the experimental procedures, which were approved by the University Committee on Research Involving Human Subjects. Postprocessing revealed that imaging missed much of the primary regions of interest in 1 subject, thereby reducing our sample to 11 .

A second group of 12 students (6 males and 6 females) from the same population was used to measure EMG responses of the flexor muscles of the right hand outside the scanner at a later date, in order to verify the efficacy of our paradigm for producing passive movement.

\section{Imaging}

During scanning, each subject lay supine in a clinical 1.5 Tesla Horizon EchoSpeed scanner from General Electric Medical Systems. The subject's head was inside a standard quadrature birdcage head coil. Foam padding limited head motion, and ear plugs reduced the impact of gradient switching noises. Functional images were acquired using a $\mathrm{T} 2 *$-weighted gradient-echo EPI sequence sensitive to blood oxygenation level-dependent (BOLD) effects (flip angle, $90^{\circ}$; TE, $45 \mathrm{msec}$; TR, $2 \mathrm{sec}$; FOV, $24 \times 24 \mathrm{~cm}$; matrix, $64 \times 64$; slice thickness, $6 \mathrm{~mm}$; voxel size, $3.75 \times 3.75 \times 6 \mathrm{~mm}$; 4 axial slices; 125 images/slice; 500 images/series). This method provides an indirect measure of neural activity, since it relies on a rise in local blood oxygen content that follows an increase in local synaptic activity. In particular, it is currently debated how f MRI signals are correlated with intracortical activity within a cortical area, as compared with the spiking output of isolated units (see Heeger \& Ress, 2002, 
for a review). This issue will be addressed when we compare our functional imaging results with those from single-unit recordings.

Our regions of interest (ROIs) were the M1, the S1, and the SMA. These areas were manually identified in each brain according to accepted anatomical landmarks. Imaging included parts of other areas potentially related to sensorimotor function (the premotor and posterior parietal cortex), but these areas were not included owing to the combination of incomplete coverage and lack of unambiguous anatomical landmarks for defining their boundaries.

Each imaging series lasted $250 \mathrm{sec}$ and included four 30 -sec periods of task performance alternating with 30 -sec periods of rest. Functional neuroimages were overlaid on a three-dimensio nal anatomical series consisting of 124 axial slices, each $1.2 \mathrm{~mm}$ thick, acquired with an SPGR pulse sequence.

\section{Task Performance}

The chosen motor task was one that could be reproducibly performed under different types or degrees of control, ranging from external control in passive movement to internal control against low and high amounts of resistance. The closest natural analogue to the task is that of a hand waving good-bye at a constant rate. In our experimental version, the subject's right palm and fingers were fixed with tape to two ends of a board hinged at the middle to allow the four fingers to flex in unison at their metacarpal joints without moving the thumb or the wrist. In all three experimental conditions, the movements consisted of flexion and extension of all four fingers together at a rate of $2 \mathrm{~Hz}$, which was guided by the switching gradients (collecting four slices with TRs of $2 \mathrm{sec}$ created pinging twice per second). Three different versions of this movement were performed, each in a different imaging series: (1) passive, in which the subject's hand was flexed by the experimenter, who moved one end of the hinged board at the specified rate, (2) voluntary low force, in which the subject executed the same movement at the same rate, with the hinged board offering little resistance to being moved, and (3) voluntary high force, in which the subject executed the same movement at the same rate, but with rubber bands attached to the hinged board in a way that noticeably increased the force requirement for making the movement. Although measurements were not taken of actual force exerted, the constant rate requirement would tend to equate the required force across subjects within each condition, given the constant physical properties of the hinged board and the rubber bands.

The order of performing the passive and the voluntary low-force tasks was counterbalanced across subjects. The voluntary high-force task was always in the last series. In the passive task, the subject was instructed to keep the fingers completely relaxed and not to assist the experimenter's movement. Verification of the absence of muscular activity in the passive task was performed with EMG recordings in a comparable group of subjects outside the scanner at a later date. In the two voluntary tasks, the subject was instructed to focus effort on the finger muscles and to try to minimize recruitment of arm and shoulder muscles. A demonstration and a brief practice preceded imaging.

An artifact of unknown origin distorted the MR signals in the voluntary high-force series in 1 subject, which reduced our sample to 10 for that series. Sample sizes for the voluntary low-force and the passive series remained at 11 .

\section{Data Analyses}

Data were prepared for by analysis using the Medical College of Wisconsin's AFNI program (Cox, Jesmanowicz, Wong, \& Hyde, 1995; http://afni.nimh.nih.gov/afni/afni_latest.shtml). After registration to correct for head motion, active voxels in the functional images were identified by correlating raw data with a convolved reference function representing the time course of the alternating periods of rest and task within an imaging series (Friston, Jezzard, \& Turner, 1994) on a voxel by voxel basis (Bandettini, Jesmanowicz, Wong, \& Hyde, 1993). Individual brain coordinates were transformed into the Talairach stereotaxic system (Talairach \& Tournoux, 1988), but the resulting large variation in the coordinates of the central sulcus led to inaccurate localization of motor cortical areas (AC-PC distance ranged from 22.5 to $28.3 \mathrm{~mm}$ in our brains, as compared with Talairach's $23.0 \mathrm{~mm}$ ). Therefore, we assigned voxels to each of our ROIs based exclusively on the basis of manual determination of their location in the untransformed images with respect to each brain's unambiguous anatomical landmarks - namely, the central sulcus, the longitudinal fissure, the anterior commissure, and the cingulate gyrus. For our purposes, this procedure was particularly important in assigning active voxels to the SI versus the MI, where the architecture of the precentral and postcentral gyri served as definitive landmarks. Assignment of voxels to the SMA was based on the following anatomical landmarks: (1) located no more than 3 voxels lateral to the midline, (2) between the central sulcus and the anterior commissure, and (3) superior to the cingulate gyrus. There was no overlap between the SMA and the M1, using these criteria (Figure 1).

\section{Criterion of Activation}

The significance level for determining activation was set at $p \leq$ $10^{-5}$, since correlations were calculated for $\sim 3,000$ voxels in each brain. Because each voxel's correlation was based on a time series of 125 images, this meant that correlations were statistically significant down to a value of +.35 . However, as further protection against Type I error, we added the additional requirement that there be a cluster of at least two contiguous voxels, in or out of plane, orthogonal or diagonal, that met this criterion within each ROI before declaring that an ROI contained activated tissue. Center of activation within each region was determined by the location of the cluster with the highest correlation value above +.35 .

After locating all the activated clusters, we determined the location of the single best-responding cluster for each task. This best cluster was defined as the cluster, regardless of ROI or hemisphere, containing the pair of contiguous voxels whose activity was most closely correlated with the task reference function. The magnitude of the best cluster's correlation with the reference function represents the maximum degree of fidelity between that subject's cortical activation and the time course of task activity. As such, it represents each subject's best response to a task, and it served as an indication of each subject's overall maximum responsiveness. The correlation values thus obtained ranged from $+.68\left(p<10^{-19}\right)$ to $+.91\left(p<10^{-32}\right)$, indicating both the overall robustness and the individual variability of the subjects' maximum activations in our tasks.

\section{Magnitude and Volume of Activation}

Within each ROI, we computed two measures of the quantitative changes in activation that occurred with changing task demands. The first was to determine the magnitude of activation in each task condition by calculating the mean intensity of the MR signals in the pair of voxels making up the best cluster within each ROI assessed individually. AFNI's measure of intensity weights the absolute variation in mean signal amplitude in each voxel by the degree of that voxel's correlation with the task reference function. Hence, it is a relative measure of task-related response magnitude.

The second quantitative measure of changes across task conditions was to determine the spatial extent or volume of activation within each ROI in each task condition. Volume was determined by counting all the voxels that met a criterion lower than that of the best cluster, since by definition the latter included only 2 voxels. Because of the wide range of correlation values encountered across regions, tasks, and subjects, a relative criterion was chosen. This was set at two thirds of the correlation of the best cluster in each active region for that particular subject. Although arbitrary, this criterion yielded a pool of voxels whose correlations were statistically significant and in sufficient quantity to make valid comparisons across regions and tasks. As will be seen, this subject-relative approach to setting activation criteria simultaneously accommodated the enormous range of individual variability observed in brain response and protected the experiment as a whole against Type I error in a manner as rigorous or more so than extant studies. 
Axial

Coronal

Sagittal

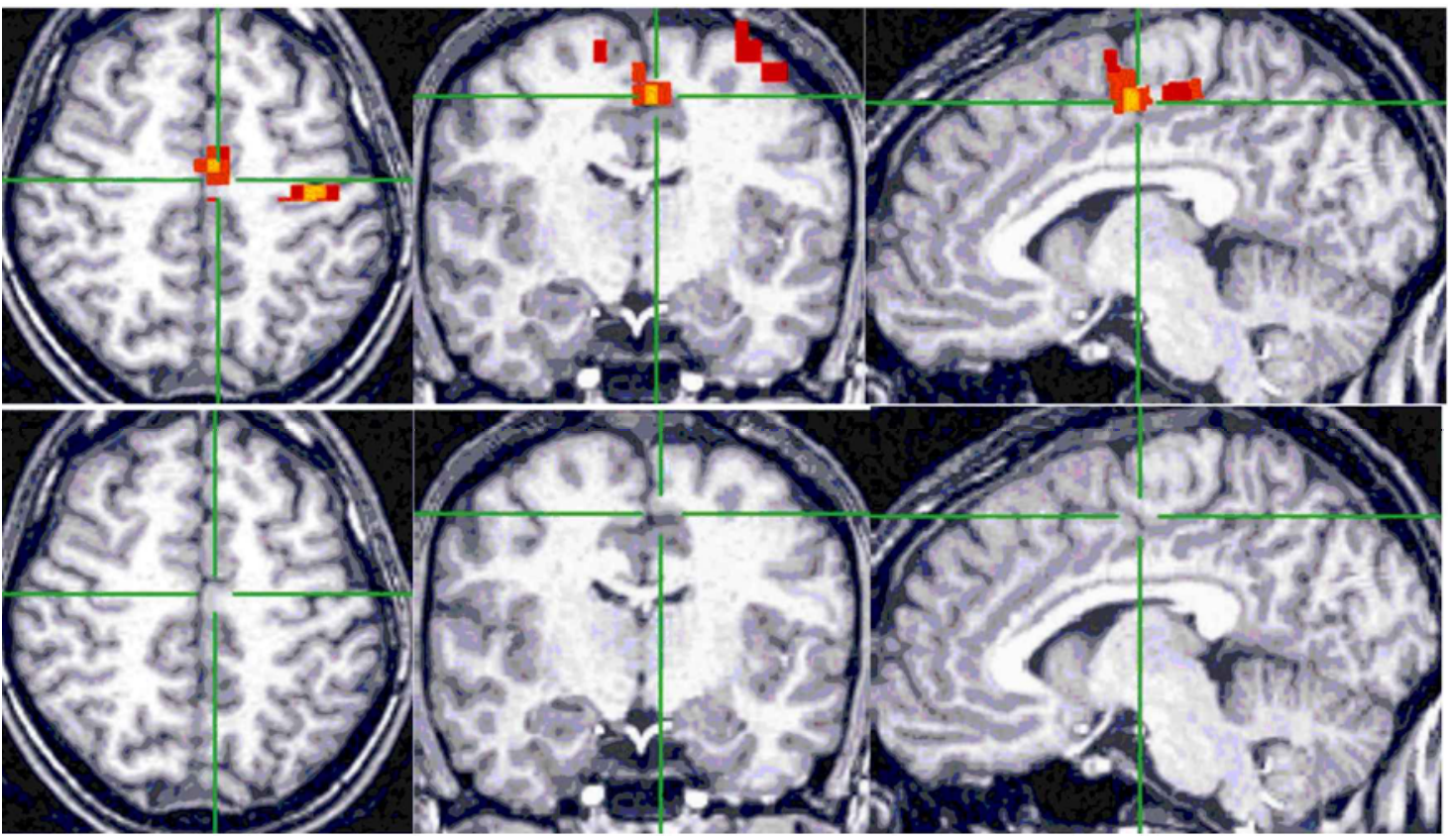

Figure 1. Brain images showing landmarks used to define our regions of interest (left hemisphere is on the right). Top and bottom rows show the same images with and without colored pixels, which represent activated voxels. Arranged in three panels from left to right, the images represent axial, coronal, and sagittal sections, in which green cross hairs are centered on the same activated cluster located bilaterally in the supplementary motor area (SMA), close to the midline. The upper image in the left panel shows a second cluster of activated voxels located more laterally in the left hemisphere. The lower image shows that the horizontal cross hair passes right along the central sulcus toward the lateral edge of the left hemisphere. However, as it approaches the midline, the central sulcus diverges sharply from the horizontal cross hair. Because of their location with respect to the central sulcus, two of the activated voxels in the second cluster are assigned to the primary motor cortex, and the other two are assigned to the somatosensory cortex. The upper and lower images in the central and right panels, representing coronal and sagittal sections, respectively, clearly show that the cross hairs are centered dorsal to the cingulate gyrus and posterior to the anterior commissure, landmarks for the SMA. These images are from a subject performing the voluntary low-force hand movement.

Absolute numerical values obtained for intensity and number of active voxels are difficult to compare across subjects and brain regions, owing to variations in baseline signal strength. For this reason, it is not possible to say whether a response in one region or subject is greater than what is observed in another. Therefore, paired $t$ tests were used to evaluate the significance of the changes in each subject's various cortical regions individually, as the type or degree of control was changed. On the basis of preliminary studies (Bernard et al., 1999; Bernard et al., 1995), our hypothesis was that as motor control ranged from purely passive to voluntary against two levels of resistance, the magnitude of response and the volume of activation would increase in each ROI.

\section{EMG Recordings}

In order to verify the lack of voluntary muscular activity in the passive movement task, EMG recordings were performed at a later date outside the scanner in a conventional laboratory setting. A pair of surface electrodes was positioned on the right forearm of each subject lying supine on a cot. A ground electrode was fixed to the dorsum of the left hand. The electrode leads fed into a preamplifier $(100 \times)$. The signal was then digitized at $1 \mathrm{kHz}$ and processed by a MacLab physiological recording system (AD Instruments, Dover, $\mathrm{NH}$ ). During task performance, the subjects' fingers were fixed to a hinged board, and movements were performed in exactly the same manner and sequence, and under the same instructions, as in the fMRI experiments.

\section{RESULTS}

\section{Best Cluster for Each Task}

The location of the single best-responding cluster for each task, independently of ROI, was always in the contralateral hemisphere. Across subjects and tasks, it was more likely to be in the $\mathrm{S} 1$ than in the M1, but never in the SMA. There was little difference in the degree to which the best clusters in the $\mathrm{S} 1$ and the best clusters in the M1 correlated with rhythmic hand movements, regardless of the type or degree of control that was required. Best-cluster correlation values ranged from +.68 to $+.91\left(10^{-38}<\right.$ $\left.p<10^{-16}\right)$ with a mean of $+0.81 \pm .6\left(p<10^{-26}\right)$. (For discussions of the problem of determining statistical significance in f MRI time series data, see Baker et al., 1994; Bullmore et al., 1996; Friston et al., 1994).

\section{Individual ROIs for Each Task}

Contralateral activation was observed in the S1, M1, and SMA in all the subjects in all three tasks (Figure 2). Ipsilateral activation was less common overall, and its distribution across ROIs varied from subject to subject. It was observed most often in the SMA, ranging from 7 sub- 
jects in the passive task to 9 in both voluntary tasks. In the M1, it was observed in all but 1 subject in the voluntary tasks, but only in 3 subjects in the passive task. Finally, ipsilateral activation was observed least often in the S1, ranging from 2 to 4 subjects across the three tasks.

The best-responding cluster within both the $\mathrm{S} 1$ and the M1 was contralateral to the moving hand in all the subjects in all three tasks, but in the SMA laterality was more variable. The SMA's best cluster was ipsilateral in 3 subjects in one or two of the tasks. Across all regions and tasks, correlation values of the best cluster ranged from +.38 to $+.91\left(10^{-38}<p<10^{-5}\right)$, with a mean across subjects and conditions of $+.74 \pm .11\left(p<10^{-22}\right)$. Generally, correlation values for ipsilateral activation were lower than for the contralateral (the only exceptions occurred in the SMA).

We should note that the mean correlation of +.74 across subjects, tasks, and ROIs corresponds to an alpha level considerably more stringent than those chosen as fixed statistical thresholds in the published studies of voluntary movement we have reviewed, and even the lowest value of +.38 easily meets standardly accepted criteria regarding choice of alpha level. Thus, our subject-relative approach to determining activation criteria takes account of individual differences while achieving high levels of protection against Type 1 error for the experiment as a whole.

\section{Magnitude and Volume of Activation and Sensitivity to Force}

As compared with passive movement, the best clusters in all three regions showed a significant increase in response magnitude during the low-force task (Table 1). However, a further increase in mean signal intensity during the high-force task was observed only in the S1.
The pattern of change in spatial extent or volume of activation was more complicated. On the basis of the criterion of two thirds of the best-cluster correlations, there was a greater number of activated voxels during voluntary low-force movement than during passive movement in the SMA, but only a trend in that direction in the M1 and no significant increase in the S1 (Table 2). In the high-force task, there was a significant increase in the M1 and the S1 relative to low force, but not in the SMA. Figure 3 illustrates the pattern of a general but varying increase in magnitude and volume of cortical activation across tasks in 1 subject.

\section{EMG Responses}

There was no significant change in muscle electrical activity between the resting and movement conditions during the passive movement task (Figure 4). Only 1 subject showed even a measurable increase, and this increase was small, as compared with the voluntary tasks. All the subjects showed large increases $(p=.0001)$ during each of the voluntary tasks (Table 3; Figure 4). Thus, our method of contrasting active versus passive movements appears to be effective.

\section{DISCUSSION}

In the present study, we used f MRI to investigate the changes in cortical activation that occurred when the same physical movement was performed under three different conditions that varied in their control requirements. The chosen task was a simple rhythmic flexion of the fingers of the right hand performed first by an external agent (the passive condition) and then by the subject against low and high resistance (the voluntary low-force and voluntary high-force conditions). Although our conditions are de-

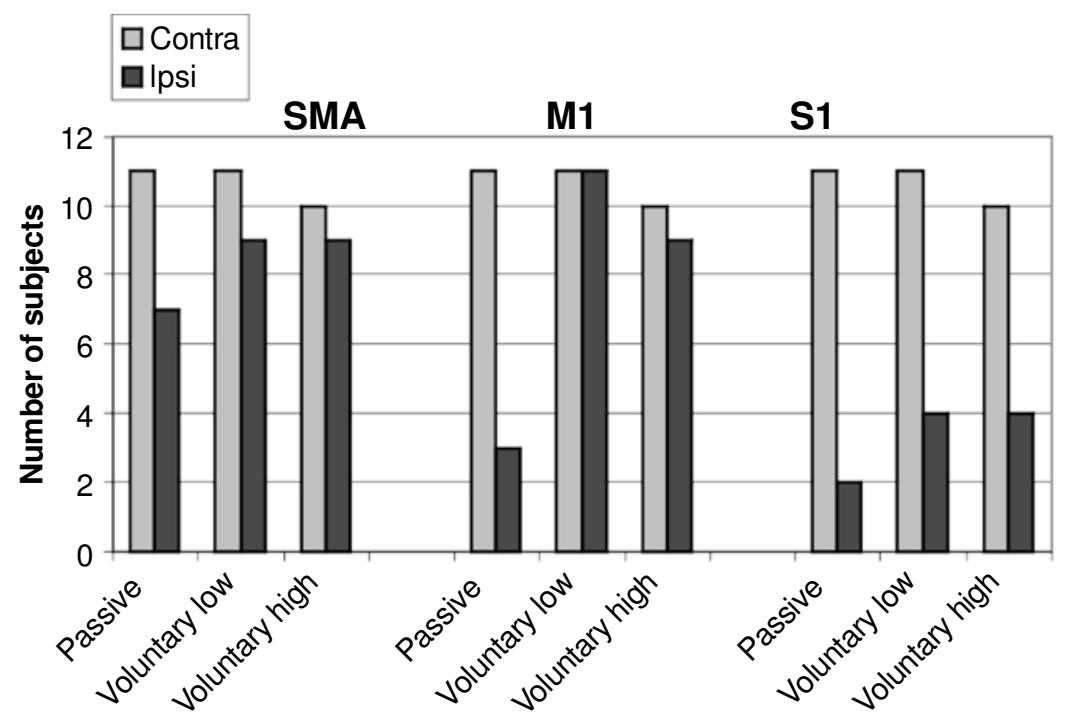

Figure 2. Number of subjects in which activation was observed in our regions of interest during the performance of a hand movement task under three levels of voluntary control. Maximum $N=11$ for passive and voluntary low force, and maximum $N=10$ for voluntary high force. SMA, supplementary motor area; M1, primary motor cortex; S1, somatosensory cortex. 
Table 1

Response Characteristics of the Best Clusters in Each Cortical Area

\begin{tabular}{|c|c|c|c|c|c|c|c|c|c|c|c|c|}
\hline \multirow[b]{3}{*}{ Type of Movement } & \multicolumn{4}{|c|}{ SMA } & \multicolumn{4}{|c|}{ M1 } & \multicolumn{4}{|c|}{ S1 } \\
\hline & \multicolumn{2}{|c|}{$\begin{array}{l}\text { Maximum } \\
\text { Correlation }\end{array}$} & \multicolumn{2}{|c|}{$\begin{array}{c}\text { Signal } \\
\text { Intensity }\end{array}$} & \multicolumn{2}{|c|}{$\begin{array}{c}\text { Maximum } \\
\text { Correlation }\end{array}$} & \multicolumn{2}{|c|}{$\begin{array}{c}\text { Signal } \\
\text { Intensity }\end{array}$} & \multicolumn{2}{|c|}{$\begin{array}{l}\text { Maximum } \\
\text { Correlation }\end{array}$} & \multicolumn{2}{|c|}{$\begin{array}{l}\text { Signal } \\
\text { Intensity }\end{array}$} \\
\hline & $M$ & $S D$ & $M$ & $S D$ & $M$ & $\overline{S D}$ & $M$ & $S D$ & $\bar{M}$ & $S D$ & $M$ & $S D$ \\
\hline Passive & .58 & \pm .12 & .314 & \pm .115 & .76 & \pm .07 & .608 & \pm .233 & .79 & \pm .06 & .561 & \pm .099 \\
\hline Voluntary low force & .64 & \pm .07 & .464 & $\pm .144^{*}$ & .79 & \pm .06 & .729 & $\pm .288 \dagger$ & .79 & \pm .09 & .647 & $\pm .183 \dagger$ \\
\hline Voluntary high force & .69 & \pm .09 & .492 & \pm .159 & .81 & \pm .06 & .791 & \pm .453 & .81 & \pm .08 & .695 & $\pm .186+$ \\
\hline
\end{tabular}

*Voluntary low force $>$ passive $(p<.02)$. †Voluntary low force $>$ passive $(p<.05)$. †Voluntary high force $>$ voluntary low force $(p<.02)$.

picted as a progressive increase in degree of control, the difference between passive and voluntary movement may also be viewed as qualitative and the difference between the two voluntary movements as quantitative. This provides an additional context for interpreting some of our results.

Analysis focused on three anatomically defined ROIs in each cortical hemisphere: the SMA, the M1, and the S1. The first major finding was that each cortical region was activated contralaterally in all three conditions in each subject, whereas ipsilateral activation differed in each region for each condition and was less consistent across subjects in any condition (Figure 2). Taken separately, each of these observations is supported by one or more of the previously described electrophysiological and functional imaging studies. Taken together, however, our findings present a new picture of how the SMA, the M1, and the S1 jointly, yet differentially, contribute to the integrated control of movement. This is primarily due to differences in the extent of ipsilateral activation in each region.

The SMA stands out from the other two regions by having the largest prevalence of ipsilateral activation in all three conditions. This would be in keeping with its classical planning role, except for the notable extension of that concept to include passive movement. In the latter case, its activity might consist in monitoring the movement and inhibiting any undesired voluntary cooperation, as was required in our study. The fact that ipsilateral activation, especially outside of the SMA, was highly variable across subjects and conditions illustrates both the extent of individual variability in cortical implementation of task control and the value of our approach to data analysis for tracking individual differences in cortical function.
These conclusions would be problematic without the evidence from EMG recordings that our task paradigms produced an effective contrast between active and passive movements. There was no indication from muscle activity that the subjects in the passive condition were mirroring or helping with the movement being performed by passive manipulation. With that evidence in hand, it does appear warranted to expand the notion of planning in the SMA to include the sorts of monitoring and regulation processes required to ensure passivity.

In contrast to the ipsilateral SMA's approximately equivalent likelihood of involvement in all three task conditions, the M1 showed a sharp increase in the number of subjects who showed ipsilateral activation in the two voluntary tasks, as compared with passive movement. Thus, whereas the ipsilateral SMA appears to be needed to ensure passivity, as well as to implement action, the role of the ipsilateral M1 appears to be more restricted to implementing action.

Despite its differential ipsilateral involvement, the M1 showed no change in the prevalence of contralateral activation across the three conditions. That is, the change in M1's execution-related activation was entirely accounted for by ipsilateral recruitment. This finding elaborates the M1's role in the execution of movement, showing that voluntarily controlled movement is dependent on the ipsilateral M1 over and above the activation of the contralateral M1 that can result from passive movement alone.

The $\mathrm{S} 1$ behaved quite differently from the other regions in having the lowest number of subjects showing ipsilateral activation in any of the conditions. In addition, it was the only region to show a significant further increase in response magnitude (Table 1) and the largest and most

Table 2

Volume of Activation at Two Thirds Maximum Correlation in Each Cortical Area

\begin{tabular}{|c|c|c|c|c|c|c|c|c|c|c|c|c|}
\hline \multirow[b]{3}{*}{ Type of Movement } & \multicolumn{4}{|c|}{ SMA } & \multicolumn{4}{|c|}{ M1 } & \multicolumn{4}{|c|}{ S1 } \\
\hline & \multicolumn{2}{|c|}{$\begin{array}{l}\text { Two-Thirds } \\
\text { Maximum } \\
\text { Correlation }\end{array}$} & \multicolumn{2}{|c|}{$\begin{array}{l}\text { Mean No. } \\
\text { of Voxels }\end{array}$} & \multicolumn{2}{|c|}{$\begin{array}{l}\text { Two-Thirds } \\
\text { Maximum } \\
\text { Correlation } \\
\end{array}$} & \multicolumn{2}{|c|}{$\begin{array}{l}\text { Mean No. } \\
\text { of Voxels }\end{array}$} & \multicolumn{2}{|c|}{$\begin{array}{l}\text { Two-Thirds } \\
\text { Maximum } \\
\text { Correlation }\end{array}$} & \multicolumn{2}{|c|}{$\begin{array}{l}\text { Mean No. } \\
\text { of Voxels }\end{array}$} \\
\hline & $M$ & $S D$ & $M$ & $S D$ & $M$ & $S D$ & $M$ & $S D$ & $M$ & $S D$ & $M$ & $S D$ \\
\hline Passive & .38 & \pm .08 & 16.3 & \pm 12.4 & .51 & \pm .05 & 14.8 & \pm 8.0 & .52 & \pm .04 & 16.9 & \pm 7.2 \\
\hline Voluntary low force & .42 & \pm .05 & 23.8 & $\pm 14.5^{*}$ & .52 & \pm .04 & 18.6 & $\pm 8.4 \dagger$ & .53 & \pm .06 & 20.5 & \pm 10.1 \\
\hline Voluntary high force & .47 & \pm .06 & 26.6 & \pm 11.5 & .54 & \pm .04 & 23.8 & $\pm 10.4 \ddagger$ & .49 & \pm .17 & 29.4 & $\pm 13.3 \S$ \\
\hline
\end{tabular}

*Voluntary low force $>$ passive $(p<.02)$. †Voluntary low force $>$ passive $(.05<p<.10) . \quad$ \$Voluntary high force $>$ voluntary low force $(p<$

$.04)$. $\S$ Voluntary high force $>$ voluntary low force $(p<.008)$. 
Passive

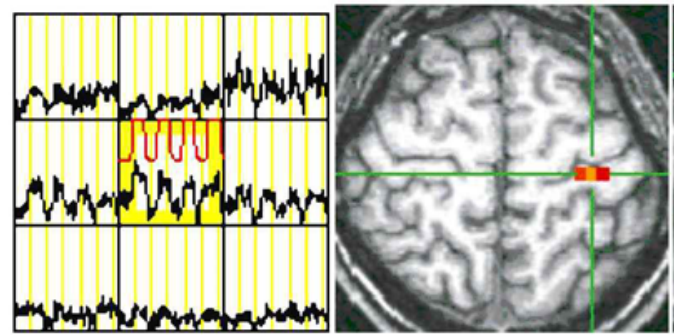

Hand Movements

Voluntary low force
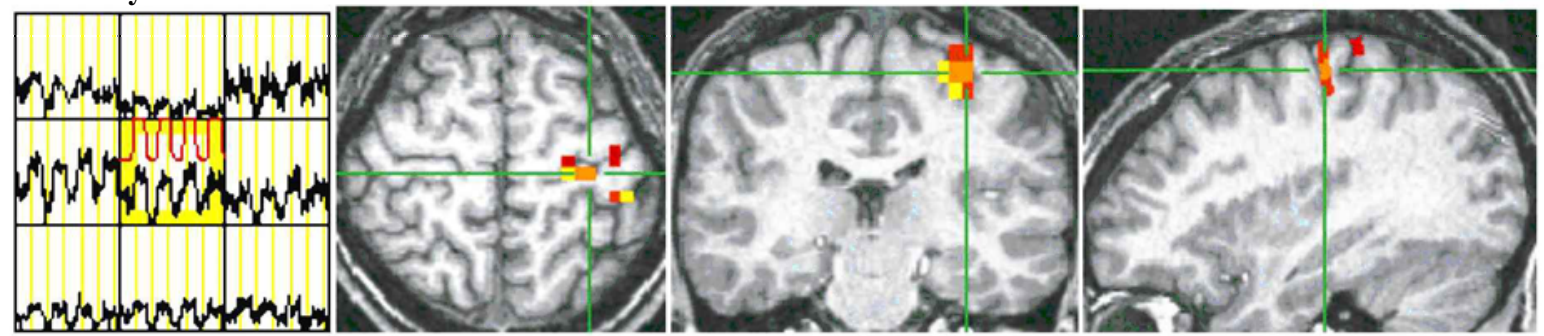

Voluntary high force
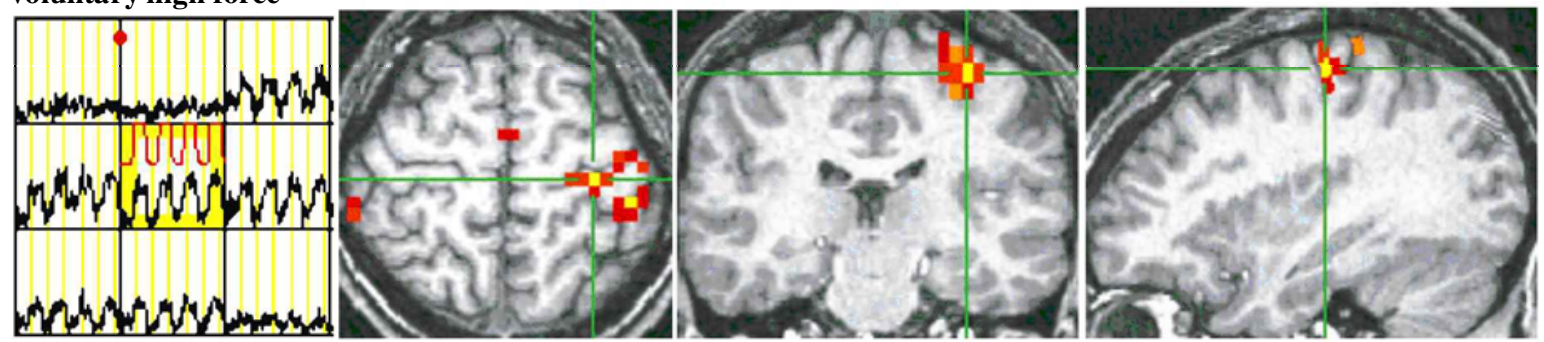

Figure 3. Change in magnitude and volume of activation as hand movement increased from purely passive to voluntary against low resistance to voluntary against high resistance. Left panel in each row displays a $3 \times 3$ array of graphs, each representing time series of MR signals in the corresponding voxels shown in the axial brain image on the right. The central graph in each array also shows the reference waveform (in red) used to perform the correlation analyses. Cross hairs are centered on the most highly correlated voxel of the best cluster, whose time series is shown in the central graph. Colored pixels represent voxels whose correlation with the task reference function was equal to or greater than two thirds that of the best cluster. Magnitude of signal change is coded yellow $>$ orange $>$ red. The best cluster remained consistently located posterior to the central sulcus, in the contralateral somatosensory cortex (S1), in this subject. Other activated clusters can be seen in the contralateral primary motor cortex, the bilateral supplementary motor area, the ipsilateral $\mathrm{S} 1$, and the posterior parietal area (not analyzed in this study).

significant increase in volume of activation (Table 2) in the voluntary high-force condition, relative to the low-force condition. These observations fit closely with the S1's role of providing feedback for the ongoing changes occurring in the contralateral hand during all three conditions.

The qualitative character of the difference between passive and voluntary movements is illustrated by the sharp increase and widespread presence of ipsilateral activation in the M1 and, to a lesser extent, the SMA, but not in the $\mathrm{S} 1$, during voluntary movement, as compared with passive movement. In addition, all three regions showed significant increases in signal intensity during the voluntary low-force task, but only the $\mathrm{S} 1$ showed a further increase in the high-force task (Table 1). To summarize, then, active control is characterized by a shift in the pattern of activation toward greater involvement of the ipsilateral motor cortex, whereas increased force is characterized by increased activation of the contralateral somatosensory cortex (Figure 2).

\section{Comparison Between fMRI Activation and Electrophysiological Activity}

Our finding of activation in the SMA, M1, and S1 during passive movement corresponds to that of electrophysiological studies in which cells in the SI, MI, and SMA are reported to fire in response to passive movement in the awake monkey (Fetz et al., 1980; Flament \& Hore, 1988; Soso \& Fetz, 1980; Tanji, 1994).The finding of activation sites in the ipsilateral SMA, MI, and SI during movement is intriguing in view of the anatomical and physiological data obtained in nonhuman primates. Only a small proportion of cells in the MI, SI, and SMA are responsive to ipsilateral stimulation in the awake behaving monkey (Tanji, Okano, \& Sato, 1988). Anatomically, some fibers do descend to the spinal cord without crossing in the pyramid, but these projections innervate proximal musculature (Kuypers \& Brinkman, 1970). Sensorimotor neural circuits connecting ipsilateral and contralateral cortical hand representation areas are indirect. Although it is well 


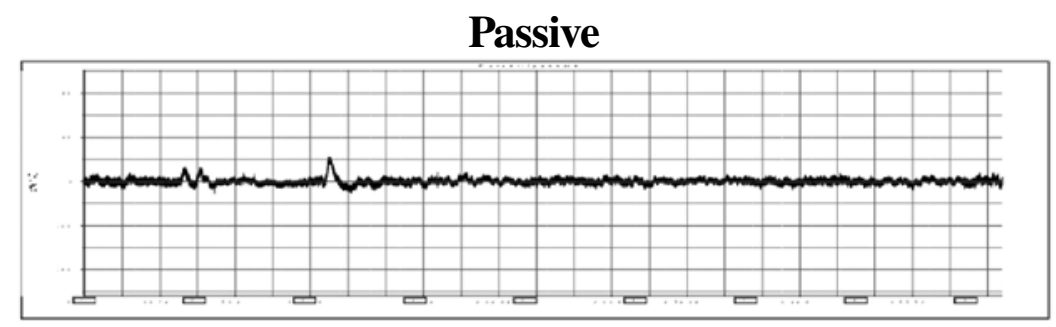

Voluntary low force

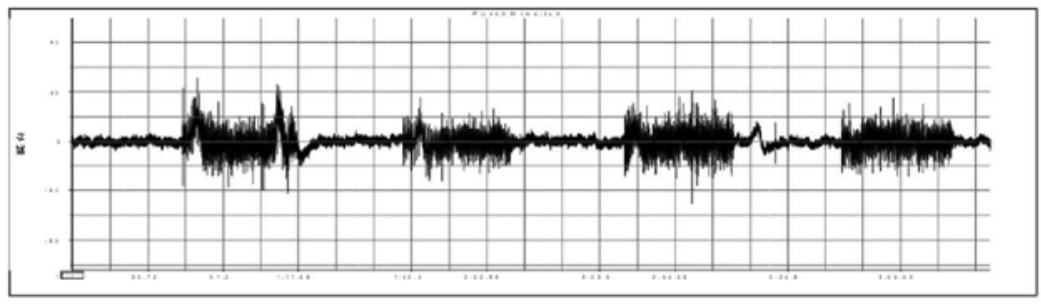

Voluntary high force

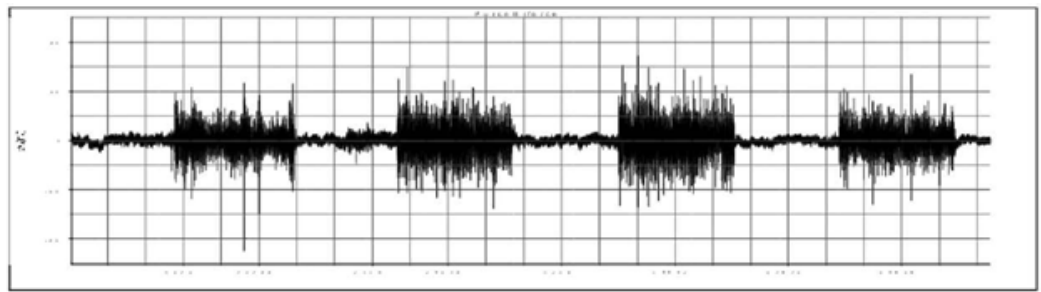

Figure 4. EMG recordings from the finger flexors in the right forearm of a subject performing our hand movement paradigm. Each record shows four cycles of finger flexion, each consisting of 30 sec of rest, followed by 30 sec of activity. Timing was the same in each condition, and bursts of muscle activity can be clearly seen in the two lower traces. The upper trace illustrates an almost complete lack of muscular activity during the passive movement condition.

known that widespread regions of the MI and the SI maintain homotopic interhemispheric connections, the hand representation is a notable exception (Gould, Cusick, Pons, \& Kaas, 1986; Jenny, 1979; Jones, Coulter, \& Wise, 1979; Jones \& Hendry, 1980; Killackey, Gould, Cusick, Pons, \& Kaas, 1983). Therefore, ipsilateral activation may be a result of commissural corticocortical projections from the hand representation to nonhomotopic regions in the MI and the SI (Jenny, 1979; Jones et al., 1979; Jones $\&$ Hendry, 1980) or by way of intrahemispheric corticocortical projections to the SMA (Jones et al., 1978). Finally, the SMA maintains robust bilateral projections and projects back to the MI and the SI (Luppino, Matelli, Camarda, \& Rizzolatti, 1993; Muakkassa \& Strick, 1979).
This inter- and intrahemispheric sensorimotor connectional network may provide the substrate for the ipsilateral activation we observed in the SMA and the M1 and, to a much lesser extent, in the S1 during voluntary movement. It is of particular interest that we observed bilateral activation of the SMA under all movement conditions, since the SMA is the region with robust bilateral projections.

Activation of the motor areas SMA and M1 during a purely passive movement highlights the different type of information provided by functional imaging, whether PET or fMRI, as compared with isolated single-unit recordings. The two functional imaging methods detect signals arising because of blood flow changes in response to the

Table 3

EMG Responses (in $\mu \mathrm{V}$ ) of Finger Flexor Muscles

\begin{tabular}{|c|c|c|c|c|c|c|}
\hline \multirow[b]{2}{*}{ Type of Movement } & \multicolumn{2}{|c|}{ Rest } & \multicolumn{2}{|c|}{ Movement } & \multicolumn{2}{|c|}{ Movement/Rest } \\
\hline & $M$ & $S D$ & $M$ & $S D$ & $M$ & $S D$ \\
\hline Passive & 34.2 & \pm 12.5 & 35.8 & \pm 12.7 & 1.1 & \pm 0.2 \\
\hline Voluntary low force & 34.9 & \pm 15.4 & 73.2 & $\pm 27.1 *$ & 2.4 & \pm 0.8 \\
\hline Voluntary high force & 36.9 & \pm 13.4 & 91.6 & $\pm 39.4 *$ & 2.7 & \pm 1.3 \\
\hline
\end{tabular}

* Movement $>$ rest $(t=5.53, p=.0001$, one-tailed). 
metabolic demands of synaptic activity. In the case of fMRI, BOLD signals arise from changes in local blood oxygen content. However, the exact relationship between neural activity, hemodynamics, and f MRI signals has not yet been clearly established. Nevertheless, most studies support the assumption that $\mathrm{fMRI}$ signals are proportional, in some way, to the local average neural activity (see Heeger \& Ress, 2002, for a review). One of the issues is that $\mathrm{fMRI}$ is correlated in various degrees with different measures of neuronal activity, single- and multi-unit spiking activity, and local field potentials. The latter leads to the possibility that fMRI reflects the input and intracortical processing of a given brain area more than does its spiking output. Thus, as Raichle (2000) emphasized, a local increase in inhibitory activity is just as likely to increase blood flow and the $\mathrm{f}$ MRI BOLD signal as is a local increase in excitatory activity.

It is possible that a network of inhibition acts upon the motor pyramidal cells (Beierlein, Gibson, \& Connors, 2000) during passive movement, since, in many respects, even though the movement is being represented in relevant brain regions, motor output must be prevented. The f MRI activation could then represent a net inhibitory effect on these same output neurons, imposed by increased activity in inhibitory interneurons. If this were so, it would not be surprising to observe robust activation in cortical motor and planning areas during a passive movement, in which voluntary production of the motor action may actually need to be inhibited, rather than merely being absent. Similarly, the overall increase in activation seen in both voluntary tasks would be due to a total increase in presynaptic input and intracortical processing, both inhibitory and excitatory, with the amount of output activity correlated with the degree of control required. This would yield a more complex picture of brain activity, in which it would be important to take into account the interplay between inhibitory and excitatory events in order to understand this and, perhaps, other cognitive functions. As was pointed out by Georgopoulos (2000), fMRI and neurophysiological methods are complementary and, together, can provide a more complete view of the neural mechanisms of behavior.

\section{Sensitivity to Force and the Interpretation of Magnitude and Volume of Activation}

Previous imaging studies in which the effect of force on cortical activation has been evaluated have produced mixed results. Although some studies have reported no force-sensitive changes (Ludman et al., 1996; Thickbroom et al., 1999), others, such as ours, have shown increased cortical activation as a function of force (Dettmers et al., 1995; Thickbroomet al., 1998). However, since those analyses did not attempt to distinguish the MI from the SI, it is difficult to directly compare their results with our own. In view of the known variability in localizing cortical areas using standardized Talairach coordinates (Grosbras, Lobel, VandeMoortele, LeBihan, \& Betholz, 1999), our own analysis initially determined the location of an activated cluster with respect to the central sulcus, using untransformed images. Thus, in our study, MI and SI activation was clearly differentiated.

It is well documented that neurons primarily in the MI (Ashe, 1997; Cheney \& Fetz, 1980; Evarts et al., 1983; Georgopoulos et al., 1992; Hepp-Reymond et al., 1978), but also in the SI (Wannier, Maier, \& Hepp-Reymond, 1991), are sensitive to movement parameters such as force (Cheney \& Fetz, 1980; Evarts, 1968; Hepp-Reymond et al., 1978). However, it is unclear how these characteristics of neuronal populations may correspond to the hemodynamic changes we observed in the MI and the SI. The increased magnitude of activation in the best cluster as more control was required suggests an analogy with the increased firing rate of individual neurons seen when there is an increase in force of contraction in a motor unit of skeletal muscle. Similarly, the increase in spatial extent or volume of activation suggests a comparison with the recruitment of spinal motor neurons as force of muscle contraction is increased. However, greater magnitude of activation within a voxel may arise both from the spread of activation to other neurons within the voxel and from increased firing among the same neurons. Also, the spread of activation to new voxels may result from the spread of inhibitory, as well as excitatory, synaptic activity and, thus, does not fully correspond to spinal motor neuron recruitment. Only cellular studies could yield such a detailed analysis. Combining such findings with those of functional imaging studies would enrich our understanding of cognition and behavior.

\section{REFERENCES}

Alexander, G. E., \& Crutcher, M. D. (1990). Preparation for movement: Neural representation of intended direction in three motor areas in the monkey. Journal of Neurophysiology, 64, 133-150.

Ashe, J. (1997). Force and the motor cortex. Behavioural Brain Research, 87, 255-269.

Baker, J. R., Weisskoff, R. M., Stern, C. E., Kennedy, D. N., Jiang, A., Kwong, K. K., Kolodny, L. B., Davis, T. L., Boxerman, J. L., BuchBinder, B. R., Wedeen, V. J., Belliveau, J. W., \& Rosen, B. R. (1994, August). Statistical assessment of functional MRI signal change. Paper presented at the Annual Meeting of the International Society for Magnetic Resonance in Medicine, San Francisco.

Bandettini, P. A., Jesmanowicz, A., Wong, E. C., \& Hyde, J. S. (1993). Processing strategies for time-course data sets in functional MRI of the human brain. Magnetic Resonance in Medicine, 30, 161-173.

Bandettini, P. A., \& Ungerleider, L. G. (2001). From neuron to BOLD: New connections. Nature Neuroscience, 4, 864-866.

Beierlein, M., Gibson, J. R., \& Connors, B. W. (2000). A network of electrically coupled interneurons drives synchronized inhibition in neocortex. Nature Neuroscience, 3, 904-910.

Bernard, R. A., Carr, T. H., Goran, D. A., McFarlane, D., Cooper, T. G., \& Potchen, E. J. (1999, April). Activity in sensorimotor cortex during simple hand movements: Comparing passive experience of motion to endogenous control of action. Paper presented at the Cognitive Neuroscience Society, Washington, DC.

Bernard, R. A., Goran, D. A., Cooper, T. G., Meyer, R. A., Egger, T. J., Backus, M. A., Putnam, C. M., Paonessa, P. D., Carr, T. H., \& Potchen, E. J. (1995, August). Cortical localization of somatic sensory and motor responses: A functionalMRI study. Paper presented at the 3rd Annual Meeting of the International Society for Magnetic Resonance in Medicine, Nice.

Bullmore, E., Brammer, M., Williams, S. C. R., Rabe-Hesketh, S., Janot, N., David, A., Mellers, J., Howard, R., \& Sham, P. (1996). 
Statistical methods of estimation and inference for functional MR image analysis. Magnetic Resonance in Medicine, 35, 261-277.

Cheney, P. D., \& Fetz, E. E. (1980). Functional classes of primate corticomotoneuronal cells and their relation to active force. Journal of Neurophysiology, 44, 773-791.

Colebatch, J. G., Deiber, M.-P., Passingham, R. E., Friston, K. J., \& FRACKOWIAK, R. S. J. (1991). Regional cerebral blood flow during voluntary arm and hand movements in human subjects. Journal of Neurophysiology, 65, 1392-1401.

Cox, R. W., Jesmanowicz, A., Wong, E. C., \& Hyde, J. S. (1995). Real-time functional magnetic resonance imaging. Magnetic Resonance in Medicine, 33, 230-238.

Deiber, M.-P., Passingham, R. E., Colebatch, J. G., Friston, K. J., Nixon, P. D., \& Frackowiak, R. S. J. (1991). Cortical areas and the selection of movement: A study with positron emission tomography. Experimental Brain Research, 84, 393-402.

Dettmers, C., Connelly, A., Stephan, K. M., Turner, R., Friston, K. J., Frackowiak, R. S., \& Gadian, D. G. (1996). Quantitative comparison of functional magnetic resonance imaging with positron emission tomography using a force-related paradigm. NeuroImage , 4, 201-209.

Dettmers, C., Fink, G. R., Lemon, R. N., Stephan, K. M., PassingHAM, R. E., \& FRACKOWIAK, R. S. J. (1995). Relation between cerebral activity and force in the motor areas of the human brain. Journal of Neurophysiology, 74, 802-815.

Donoghue, J. P., Sanes, J. N., Hatsopoulos, N. G., \& GaAl, G. (1998). Neural discharge and local field potential oscillations in primate motor cortex during voluntary movements. Journal of Neurophysiology, 79, 159-173.

EvarTs, E. V. (1968). Relation of pyramidal tract activity to force exerted during voluntary movement. Journal of Neurophysiology, 31, $14-27$

Evarts, E. V., Fromm, C., Kroller, J., \& Jennings, V. A. (1983). Motor cortex control of finely graded forces. Journal of Neurophysiology, 49, 1199-1215.

EvarTs, E. V., \& TANJI, J. (1974). Gating of motor cortex reflexes by prior instruction. Brain Research, 71, 479-494.

Fetz, E. E., Finocchio, D. V., BaKer, M. A., \& Soso, M. J. (1980). Sensory and motor responses of precentral cortex cells during comparable passive and active joint movements. Journal of Neurophysiology, 43, 1070-1089.

Flament, D., \& Hore, J. (1988). Relations of motor cortex neural discharge to kinematics of passive and active elbow movements in the monkey. Journal of Neurophysiology, 60, 1268-1284.

Friston, K. J., Jezzard, P., \& Turner, R. (1994). The analysis of functional MRI time series. Human Brain Mapping, 1, 153-171.

Galea, M. P., \& Darian-Smith, I. (1994). Multiple corticospinal neuron populations in the macaque monkey are specified by their unique cortical origins, spinal terminations, and connections. Cerebral Cortex, 4, 166-194.

GEORGopoulous, A. P. (2000). Neural mechanisms of motor cognitive processes: Functional MRI and neurophysiological studies. In M. S. Gazzaniga (Ed.), The new cognitive neurosciences (2nd ed., pp. 531-537). Cambridge, MA: MIT Press.

Georgopoulos, A. P., Ashe, J., Smy rnis, N., \& Taira, M. (1992). The motor cortex and the coding of force. Science, 256, 1692-1695.

Ghez, C., \& Krakauer, J. (2000). The organization of movement. In E. Kandel, J. H. Schwartz, \& T. M. Jessell (Eds.), Principles of neural science (pp. 653-673). New York: McGraw-Hill.

Gould, H. J., Cusick, C. G., Pons, T. P., \& KaAs, J. H. (1986). The relationship of corpus callosum connections to electrical stimulation maps of motor, supplementary motor and the frontal eye fields in owl monkeys. Journal of Comparative Neurology, 247, 297-325.

Grafton, S. T., Woods, R. P., \& Mazziotta, J. C. (1993). Within-arm somatotopy in human motor areas determined by positron emission tomography imaging of cerebral blood flow. Experimental Brain Research, 95, 172-176.

Grosbras, M.-H., Lobel, E., VandeMoortele,P.-F., LeBihan, D., \& Betholz, A. (1999). An anatomical landmark for the supplementary eye fields in human revealed with functional magnetic resonance imaging. Cerebral Cortex, 9, 705-711.
HeEger, D. J., \& Ress, D. (2002). What does f MRI tell us about neuronal activity? Nature Reviews: Neuroscience, 3, 142-151.

Hepp-Reymond, M.-C., Wyss, U. R., \& AnNer, R. (1978). Neuronal coding of force and the rate of force change in the pre-central finger region of the monkey. Experimental Brain Research, 7 (Suppl.), 315326.

Jenny, A. B. (1979). Commissural projections of the cortical hand motor area in monkeys. Journal of Comparative Neurology, 188, 137 146.

Jones, E. G. (1985). The thalamus. New York: Plenum.

Jones, E. G., Coulter, J. D., \& Hendry, S. H. (1978). Intracortical connectivity of architectonic fields in the somatic sensory, motor and parietal cortex of monkeys. Journal of Comparative Neurology, 181, 291-347.

Jones, E. G., Coulter, J. D., \& Wise, S. P. (1979). Commissural columns in the sensory-motor cortex of monkeys. Journal of Comparative Neurology, 188, 113-136.

Jones, E. G., \& HENDRY, S. H. (1980). Distribution of callosal fibers around the hand representations in monkey somatic sensory cortex. Neuroscience Letters, 19, 167-172.

Killackey,H. P., Gould, H. J., Cusick, C. G., Pons, T. P., \& KaAs, J. H. (1983). The relation of corpus callosum connections to architectonic fields and body surface maps in sensorimotor cortex of New and Old World monkeys. Journal of Comparative Neurology, 219, 384-419.

KuY PERs, H. G., \& BRINKMAN, J. (1970). Precentral projections to different parts of the spinal intermediate zone in the rhesus monkey. Brain Research, 24, 29-48.

Lemon, R. N., \& Porter, R. (1976). Afferent input to movement-related precentral neurones in conscious monkeys. Proceedings of the Royal Society of London: Series B, 194, 313-339.

Logothetis, N. K., Paulis, J., Augath, M., Trinath, T., \& OelterMANN, A. (2001). Neurophysiological investigation of the basis of the f MRI signal. Nature, 412, 150-157.

Ludman, C. N., Cooper, T. G., Ploutz-Synder, L. L., Potchen, E. J., \& MeYer, R. A. (1996). Force of voluntary exercise does not affect sensorimotor cortex activation as detected by functional MRI at $1.5 \mathrm{~T}$. NMR in Biomedicine, 9, 228-232.

Luppino, G., Matelli, M., Camarda, R, \& Rizzolatti, G. (1993). Corticocortical connections of area F3 (SMA-proper) and area F6 (pre-SMA) in the macaque monkey. Journal of Comparative Neurology, 338, 114-140.

Menon, R. S. (2001). Imaging function in the working brain with $\mathrm{f}$ MRI. Current Opinion in Neurobiology, 11, 630-636.

Mima, T., Sadato, N., Yazawa, S., Hanakawa, T., Fukuyama, H., YoneKURA, Y., \& ShIBASAKI, H. (1999). Brain structures related to active and passive finger movements in man. Brain, 122, 1989-1997.

MuakKassa, K. F., \& Strick, P. L. (1979). Frontal lobe inputs to primate motor cortex: Evidence for four somatotopically organized "premotor" areas. Brain Research, 177, 176-182.

Murray,E. A., \& Coulter, J. D. (1981). Organization of corticospinal neurons in the monkey. Journal of Comparative Neurology, 195, 339365.

Porro, C. A., Francescato, M. P., Cettolo, V., Diamond, M. E., Baraldi, P., Zuiani, C., Bassocchi, M., \& di Prampero, P. E. (1996). Primary motor and sensory cortex activation during motor performance and motor imagery: A functional magnetic resonance imaging study. Journal of Neuroscience, 16, 7688-7698.

Prud'homme, M. J., Cohen, D. A., \& Kalaska, J. F. (1994). Tactile activity in primate primary somatosensory cortex during active arm movements: Cytoarchitectonic distribution. Journal of Neurophysiology, 71, 173-181.

RAichle, M. E. (2000). The neural correlates of consciousness: An analysis of cognitive skill learning. In M. S. Gazzaniga (Ed.), The new cognitive neurosciences (2nd ed., pp. 1313-1314). Cambridge, MA: MIT Press.

Rao, S. M., Binder, J. R., Bandettini, P. A., Hammeke, T. A., Yetkin, F. Z., Jesmanowicz, A., Lisk, L. M., Morris, G. L., Mueller, W. M., Est kowski, L. D., Wong, E. C., Haughton, V. M., \& HY DE, J. S. (1993). Functional magnetic resonance imaging of complex human movements. Neurology, 43, 2311-2318.

Rausch, M., Spengler, F., \& Eysel, U. T. (1998). Proprioception acts 
as the main source of input in human S-I activation experiments: A functional MRI study. NeuroReport, 9, 2865-2868.

Rouiller, E. M., Liang, F., Babalian, A., Moret, V., \& WiesenDANGER, M. (1994). Cerebellothalamocortical and pallidothalamocortical projections to the primary and supplementary motor cortical areas: A multiple tracing study in macaque monkeys. Journal of Comparative Neurology, 345, 185-213.

SAKAI, S. T., INASE, M., \& TANJI, J. (1996). Comparison of cerebellothalamic and pallidothalamic projections in the monkey (Macaca fuscata): A double anterograde labeling study. Journal of Comparative Neurology, 368, 215-228.

SChell, G. R., \& Strick, P. L. (1984). The origin of thalamic inputs to the arcuate premotor and supplementary motor areas. Journal of Neuroscience, 4, 539-560.

Soso, M. J., \& Fetz, E. E. (1980). Responses of identified cells in postcentral cortex of awake monkeys during comparable active and passive joint movements. Journal of Neurophysiology, 43, 1090-1110.

TAlairach, J., \& Tournoux, P. (1988). Co-planar stereotaxic atlas of the human brain. Stuttgart: Thieme.

TANJI, J. (1994). The supplementary motor area in the cerebral cortex. Neuroscience Research, 19, 251-268.

TANJI, J., \& Kurata, K. (1982). Comparison of movement-related activity in two cortical motor areas of primates. Journal of Neurophysiology, 48, 633-653.

TAnjI, J., OKano, K., \& SATo, K. (1988). Neuronal activity in cortical motor areas related to ipsilateral, contralateral and bilateral digit movements of the monkey. Journal of Neurophysiology, 60, 325-343.
Thickbroom, G. W., Phillips, B. A., Morris, I., Byrnes, M. L., \& Mastaglia, F. L. (1998). Isometric force-related activity in sensorimotor cortex measured with functional MRI. Experimental Brain Research, 121, 59-64.

Thickbroom, G. W., Phillips, B. A., Morris, I., Byrnes, M. L., Sacco, P., \& Mastaglia, F. L. (1999). Differences in functional magnetic resonance imaging of sensorimotor cortex during static and dynamic finger flexion. Experimental Brain Research, 126, 431-438.

Turner, R. S., Grafton, S. T., Votaw, J. R., DeLong, M. R., \& HoffMANN, J. M. (1998). Motor subcircuits mediating the control of movement velocity: A PET study. Journal of Neurophysiology, 80, 21612176.

Wannier, T. M., Maier, M. A., \& Hepp-Reymond, M. C. (1991).Contrasting properties of monkey somatosensory and motor cortex neurons activated during the control of force in precision grip. Journal of Neurophysiology, 65, 572-589.

Weiller, C., Juptner, M., Fellows, S., Rijntjes, M., Leonhardt, G., Kiebel, S., Muller, S., Diener, H. C., \& Thilmann, A. F. (1996). Brain representations of active and passive movements. NeuroImage, 4, 105-110.

Xiong, J., Parsons, L. M., GaO, J. H., \& Fox, P. T. (1999). Interregional connectivity to primary motor cortex revealed using MRI resting state images. Human Brain Mapping, 8, 151-156.

(Manuscript received September 18, 2001; revision accepted for publication June 10, 2002.) 